\title{
Laser oscillations of whispering gallery modes in thiophene/phenylene co-oligomer microrings
}

\author{
Seiji Fujiwara, Kazuki Bando, and Yasuaki Masumoto ${ }^{a)}$ \\ Institute of Physics and Center for Tsukuba Advanced Research Alliance (TARA), University of Tsukuba, \\ Tsukuba, Ibaraki 305-8571, Japan \\ Fumio Sasaki and Shunsuke Kobayashi \\ Photonics Research Institute, National Institute of Advanced Industrial Science and Technology (AIST), \\ Tsukuba, Ibaraki 305-8568, Japan \\ Satoshi Haraichi \\ Nanoelectronics Research Institute, National Institute of Advanced Industrial Science and Technology \\ (AIST), Tsukuba, Ibaraki 305-8568, Japan \\ Shu Hotta \\ Department of Macromolecular Science and Engineering, Graduate School of Science and Technology, \\ Kyoto Institute of Technology, Kyoto 606-8585, Japan
}

(Received 2 April 2007; accepted 14 June 2007; published online 9 July 2007)

\begin{abstract}
Laser oscillation of whispering gallery modes was observed in microring structures of semiconducting thiophene/phenylene co-oligomer (TPCO) crystals at room temperature. Microring structures were formed by dry etching from thin film crystals of TPCO. The thresholds for the laser oscillation of a microring and a thin film crystal are 200 and $1400 \mu \mathrm{J} / \mathrm{cm}^{2}$ for picosecond excitation, respectively. Therefore, the threshold for the microring was reduced to $1 / 7$ of that for the thin film crystal. The dramatic reduction of threshold clearly demonstrates the importance of microcavity in making efficient organic semiconductor lasers. (C) 2007 American Institute of Physics.
\end{abstract}

[DOI: 10.1063/1.2755925]

Recently, thiophene/phenylene co-oligomers (TPCOs) have attracted much attention because they show excellent optical and transport properties at room temperature (RT). Crystals of TPCO showed spectrally narrowed emissions under high density excitation at RT, and a stimulated emission including amplified spontaneous emission (ASE) was reported. $^{1-3}$ Laser oscillation has been demonstrated as well. ${ }^{4,5}$ The stimulated resonance Raman scattering and pulselike emission with time delay and other optical amplification processes were observed. ${ }^{6-8}$ In addition, TPCO crystals exhibited electroluminescence and showed good performance of field-effect transistors at RT. ${ }^{9,10}$ Therefore, these materials are expected to be good candidates for organic laser materials.

Both the polymer organic materials and the aligned crystalline organic materials are good candidates for organic semiconductor lasers. Carrier mobility of crystalline organic materials is generally higher than that of polymers, and a very high mobility exceeding $10 \mathrm{~cm}^{2} / \mathrm{V} \mathrm{s}$ was reported in crystalline organic materials. ${ }^{11}$ So, highly crystalline organic materials are preferable to the realization of organic semiconductor lasers. Low lasing threshold is very important for the application. It is believed that confining the light into high-gain materials reduces the lasing threshold. Lasing in microcavities such as Fabry-Pérot cavities, rings, disks, and spheres has been demonstrated not only in inorganic but also organic semiconductors. ${ }^{12-17}$ Microcavities provide a strong coupling of stimulated emission into lasing modes, and consequently high $Q$ values lead to low lasing thresholds. In this letter, we reported laser oscillations of whispering gallery

${ }^{a)}$ Electronic mail: shoichi@sakura.cc.tsukuba.ac.jp modes (WGMs) in TPCO microring cavities excited by a picosecond laser.

We used 2,5-bis(4-biphenylyl)thiophene (BP1T) crystals as organic high-gain media. ${ }^{18,19}$ The fundamental optical properties and the origin of the ASE observed in BP1T single crystals at low temperature were reported previously. ${ }^{20}$ Firstly, a buffer layer $300 \mathrm{~nm}$ thick of amorphous fluorocarbon polymer, Cytop CTX804.5A (ASAHI GLASS Co., Ltd., Japan), was spin coated on a $\mathrm{SiO}_{2}$ layer $160 \mathrm{~nm}$ thick formed by thermal oxidation on the silicon (100) substrates. The buffer layer worked to suppress the crack formation during the crystallization of the BP1T amorphous layer. Secondarily, a BP1T amorphous layer $400 \mathrm{~nm}$ thick was formed on the buffer layer by the vapor deposition of BP1T microcrystals and an overlayer $300 \mathrm{~nm}$ thick of the CTX804.5A was made by spin coating to prevent desorption of BP1T during the course of its crystallization. Further thermal annealing by a hot plate formed BP1T thin film crystals at $220^{\circ} \mathrm{C}$. After annealing the BP1T film, long molecular axis of BP1T stood perpendicular to the $\mathrm{SiO}_{2}$ surface, as is shown schematically in the right inset of Fig. 1, and the transition dipole moments were nearly parallel to the long molecular axes. ${ }^{21}$

Microring structures were made by means of dry etching processes. First, a $1 \mathrm{~nm} \mathrm{Cr} / 30 \mathrm{~nm} \mathrm{Si} / 1 \mathrm{~nm} \mathrm{Cr}$ etch mask layer was deposited. After a layer of polymethylmethacrylate (PMMA), electron beam resist, OEBR-1000 (Tokyo Ohka Kogyo Co., Ltd., Japan), was spin coated, a reversal pattern was formed by the electron beam lithography [Fig. 1(a)]. Molecules of TPCO are hardly soluble in organic sovents of Cytop, and Cytop polymers to solvents of PMMA. In fact, we observed no morphology change and local delamination in cytop/BP1T/cytop films formed by successive spin coating and baking. A Si etch mask layer was formed by reactive ion 

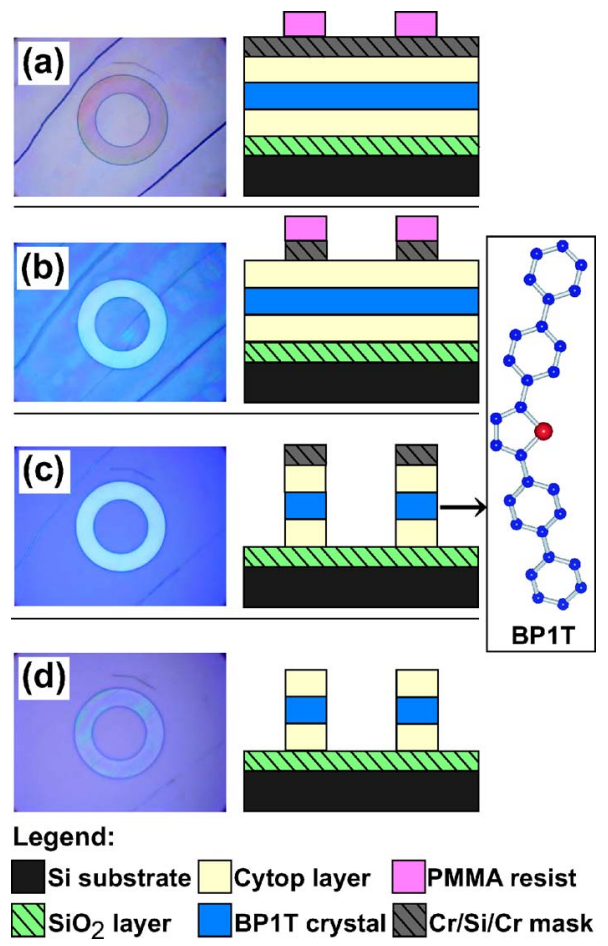

FIG. 1. (Color online) Laser microscope images and schematic fabrication procedures of BP1T microring lasers. See the right inset for the backbone model of BP1T. Here $\mathrm{C}$ atoms are represented by small circles and a $\mathrm{S}$ atom is represented by a large circle. (a) Reversal patterning process by use of the electron beam lithography. (b) Fabrication of a $\mathrm{Si}$ etch mask layer by $\mathrm{CHF}_{3} / \mathrm{SF}_{6} / \mathrm{CHF}_{3}$ RIE processes. (c) Fabrication of Cytop and BP1T crystal layers by $\mathrm{O}_{2}$ RIE. (d) Removal of a $\mathrm{Si}$ mask by $\mathrm{CHF}_{3} / \mathrm{SF}_{6} / \mathrm{CHF}_{3} \mathrm{RIE}$ processes.

etching (RIE) with the use of $\mathrm{CHF}_{3}, \mathrm{SF}_{6}$, and $\mathrm{CHF}_{3}$ gases in series [Fig. 1(b)]. The microring structures of BP1T were fabricated by means of an $\mathrm{O}_{2}$ RIE, and the remaining PMMA resist was removed [Fig. 1(c)]. After the Si mask was removed by means of the $\mathrm{CHF}_{3} / \mathrm{SF}_{6} / \mathrm{CHF}_{3}$ RIE processes [Fig. 1(d)], the microrings were formed. The thin crystalline BP1T microrings were formed on the $\mathrm{SiO}_{2}$ layer.

We observed the laser emission from BP1T microrings whose diameters are 10, 20, 30, and $40 \mu \mathrm{m}$ at RT. The excitation source was the second harmonic generation $(397 \mathrm{~nm})$ of the output from a regenerative amplifier of a Ti:sapphire laser with the pulse width of $10-15 \mathrm{ps}$ at the repetition rate of $1 \mathrm{kHz}$. The laser emission was detected by using a monochromator and a liquid-nitrogen-cooled chargecoupled device. These microrings were excited by the pump laser with an excitation area of $100 \times 100 \mu \mathrm{m}^{2}$ which is larger than the areas of microrings. Thus, each microring was excited homogeneously. The transition dipole moment of each BP1T microring is aligned to a molecule length axis and is nearly vertical to the substrate. Therefore, $p$ polarized light with the incident angle of $50^{\circ}$ was used to excite the BP1T microrings for the efficient pumping.

Figure 2 shows the lasing spectra from BP1T microrings $10,20,30$, and $40 \mu \mathrm{m}$ in diameter. The lasing spectra ranging from 2.64 to $2.70 \mathrm{eV}$ correspond to the $0-1$ vibronic band of BP1T. ${ }^{20}$ The value of $Q$ can be directly obtained from the linewidth of the resonator mode. The maximum $Q$ value of the microring was estimated to be 2200 at $2.67 \mathrm{eV}$ for a microring of $20 \mu \mathrm{m}$ in diameter.

Equally spaced peaks were observed from each microring. The mode interval becomes smaller with increasing miDownloaded 09 Jul 2007 to 130.158.105.240. Redistribution subject

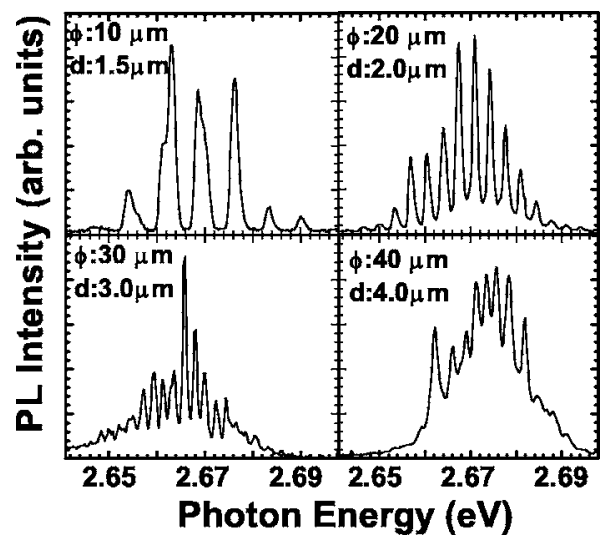

FIG. 2. Lasing spectra of BP1T microrings: (a) A diameter $\phi=10 \mu \mathrm{m}$ and a ring width $d=1.5 \mu \mathrm{m}$. (b) $\phi=20 \mu \mathrm{m}$ and $d=2 \mu \mathrm{m}$. (c) $\phi=30 \mu \mathrm{m}$ and $d=3 \mu \mathrm{m}$. (d) $\phi=40 \mu \mathrm{m}$ and $d=4 \mu \mathrm{m}$.

croring diameters because of the oscillations due to higher order modes. These mode intervals are calculated by the fast Fourier transformation, indicating the presence of a main peak given by the inverse of the period. The relationship between the mode interval and the resonator length is plotted in Fig. 3. Here the circumferential length of the microring was assumed to be the resonator length $L$. The solid line in Fig. 3 is the least-squares fitting. Luminescence peaks were separated from each other by $E_{m}=m h c / n L$, given by

$$
\Delta E_{m}=E_{m+1}-E_{m}=\frac{h c}{n_{\mathrm{eff}} L}, \quad n_{\mathrm{eff}}=\left(n+\left.E \frac{d n}{d E}\right|_{E m}\right),
$$

where $h$ is the Planck constant, $c$ is the velocity of light in vacuum, $n_{\text {eff }}$ is the effective refraction index, and $n$ is the refraction index of the gain materials. ${ }^{22}$ Thus the inverse proportion between the mode interval $\Delta E$ and the resonator length $L$ represented in Fig. 3 is a direct consequence of the presence of cavity multimodes confined as standing waves in the resonator. Consequently, we conclude that the microring lasers reflect WGMs.

The calculated WGMs were obtained by the analysis based on the finite difference time domain method. The node number of the electromagnetic field in the radial direction $n_{r}$ is assumed to be 1 or 2 for the microring diameter of $20 \mu \mathrm{m}$. Then, the node number of circumferential direction $n_{\theta}$ is $\left(n_{r}, n_{\theta}\right)=(1,243-248),(2,234-239)$ in the $0-1$ amplification line. Both TE and TM modes are allowed in the WGM so

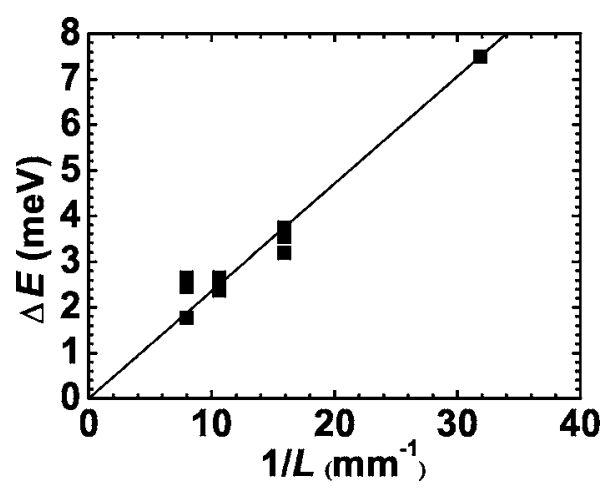

FIG. 3. Mode intervals $\Delta E$ as a function of the resonator length $L$ given by the circumferential length of the microring. The solid line is the leastsquares fitting of the mode interval vs the inverse of the length of the resonator. 


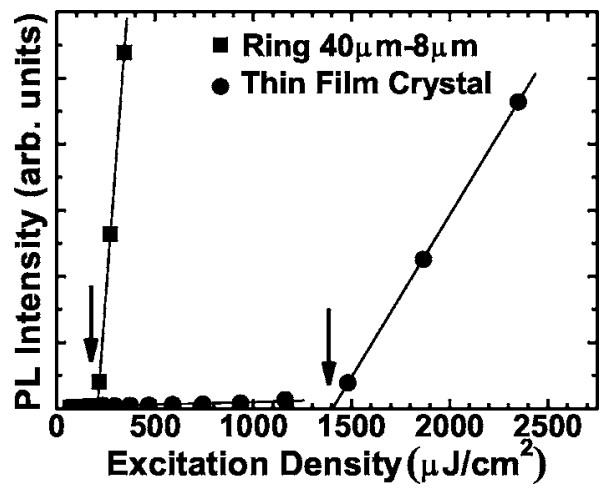

FIG. 4. Excitation density dependence of the highest emission peak intensities of a microring laser (solid square) and a thin film crystal (solid circle). The diameter of a microring laser is $40 \mu \mathrm{m}$ and the ring width is $8 \mu \mathrm{m}$. The thresholds for the laser oscillation of a microring and a thin film crystal are 200 and $1400 \mu \mathrm{J} / \mathrm{cm}^{2}$, respectively.

that the total number of the WGM's should be $6 \times 4=24$ in the $0-1$ amplification region. However, peak energies of $(1, x)$ on the TM mode give close agreement with that of $(2, x-8)$ on the TE mode. We thought that these two modes could not be resolved in the experiment. On this assumption, the total number of WGM's should be $6 \times 3=18$, which gives the good agreement with the observed 17 WGM's, as shown in Fig. 2.

Figure 4 shows the emission intensity of a BP1T thin film crystal and a microring formed on the same BP1T thin film crystal as a function of the excitation density. The typical thresholds for the laser oscillation of a BP1T microring and a BP1T thin film crystal are 200 and $1400 \mu \mathrm{J} / \mathrm{cm}^{2}$, respectively. The laser oscillation threshold for the BP1T microring could be reduced to $1 / 7$ of that for the BP1T thin film crystal by the efficient confinement of the light in the microring. That means very high-quality microring lasers could be fabricated.

In summary, we observed the laser oscillation in the 0-1 band from BP1T microrings excited by picosecond laser pulses. The observed lasing modes were successfully controlled with the change of the diameter of microrings. Ring circumferential lengths were inversely proportional to the mode interval of the laser oscillation. These results confirmed that the laser oscillations took place in WGMs. The lasing threshold for the microring was reduced to $1 / 7$ of that for the thin film crystal. Microring structure was demonstrated to be useful for the organic semiconductor laser and other optical and electronic devices.

${ }^{1}$ M. Nagawa, R. Hibino, S. Hotta, H. Yanagi, M. Ichikawa, T. Koyama, and Y. Taniguchi, Appl. Phys. Lett. 80, 544 (2002).

${ }^{2}$ M. Ichikawa, R. Hibino, M. Inoue, T. Haritani, S. Hotta, T. Kayama, and Y. Taniguchi, Adv. Mater. (Weinheim, Ger.) 15, 213 (2003).

${ }^{3}$ K. Shimizu, D. Hoshino, and S. Hotta, Appl. Phys. Lett. 83, 4494 (2003).

${ }^{4}$ M. Ichikawa, R. Hibino, M. Inoue, T. Haritani, S. Hotta, K. Araki, T. Koyama, and Y. Taniguchi, Adv. Mater. (Weinheim, Ger.) 17, 2073 (2005).

${ }^{5}$ K. Shimizu, Y. Mori, and S. Hotta, J. Appl. Phys. 99, 063505 (2006).

${ }^{6}$ H. Yanagi, A. Yoshiki, S. Hotta, and S. Kobayashi, Appl. Phys. Lett. 83, 1941 (2003).

${ }^{7}$ K. Ishikawa, F. Sasaki, S. Kobayashi, H. Yanagi, S. Hotta, and Y. Taniguchi, J. Lumin. 108, 127 (2004).

${ }^{8}$ F. Sasaki, S. Kobayashi, S. Haraichi, H. Yanagi, S. Hotta, M. Ichikawa, and Y. Taniguchi, Jpn. J. Appl. Phys., Part 2 45, L1206 (2006).

${ }^{9}$ M. Ichikawa, H. Yanagi, Y. Shimizu, S. Hotta, N. Suganuma, T. Kayama, and Y. Taniguchi, Adv. Mater. (Weinheim, Ger.) 14, 1272 (2002).

${ }^{10}$ H. Yanagi, and T. Morikawa, and S. Hotta, Appl. Phys. Lett. 81, 1512 (2002).

${ }^{11}$ V. C. Sundar, J. Zaumseil, V. Pozorov, E. Menard, R. L. Willett, T. Someya, M. E. Gershenson, and J. A. Rogers, Science 303, 1644 (2004).

${ }^{12}$ S. L. McCall, A. F. J. Levi, R. E. Slusher, S. J. Pearton, and R. A. Logan, Appl. Phys. Lett. 60, 289 (1992).

${ }^{13}$ M. Kuwata-Gonokami, K. Takeda, H. Yasuda, and K. Ema, Jpn. J. Appl. Phys., Part 2 31, L99 (1992).

${ }^{14}$ Y. Yamamoto and R. E. Slusher, Phys. Today 46, 66 (1993).

${ }^{15}$ M. Kuwata-Gonokami, R. H. Jordan, A. Dodabalapur, H. E. Katz, M. L. Schilling, and R. E. Slusher, Opt. Lett. 20, 2093 (1995).

${ }^{16}$ S. V. Frolov, M. Shkunov, Z. V. Vardeny, and K. Yoshino, Phys. Rev. B 56, 4363 (1997).

${ }^{17}$ K. J. Vahala, Nature (London) 424, 839 (2003).

${ }^{18}$ S. Hotta, H. Kimura, S. A. Lee, and T. Tamaki, J. Nondestruct. Eval. 37, 281 (2000).

${ }^{19}$ S. Hotta and M. Goto, Adv. Mater. (Weinheim, Ger.) 14, 498 (2002).

${ }^{20}$ K. Bando, T. Nakamura, Y. Masumoto, F. Sasaki, S. Kobayashi, and S. Hotta, J. Appl. Phys. 99, 013518 (2006).

${ }^{21}$ We confirmed the molecular arrangement by angle-resolved transmission spectra. With increasing the incident angle, the absorption band at $4.02 \mathrm{eV}$ grows in $p$ polarization but not in $s$ polarization. This shows that the absorption band is ascribed to the $\mathrm{H}$ band and that the transition dipole moment parallel to the long molecular axis is perpendicular to the $\mathrm{SiO}_{2}$ surface.

${ }^{22}$ J. I. Pankove, Optical Processes in Semiconductors (Dover, New York, 1971), Chap. 10, p. 222. 